\title{
Lesado medular. Estudo prospectivo de 92 casos
}

\author{
Luís Renato Mello*, Gisele Espíndola**, Fabrício Molon da Silva***, Celso Itiberê Bernardes ${ }^{\star * * *}$ \\ Serviço de Neurocirurgia do Hospital Santa Isabel. Blumenau, SC
}

\section{RESUMO}

O objetivo do trabalho foi avaliar de forma seriada a função neurológica de pacientes com traumatismo de coluna vertebral e medula espinhal, correlacionando-a à reabilitação neurológica durante a internação e a terapêutica estabelecida. Foram estudados, de forma prospectiva, 92 pacientes portadores de traumatismo de coluna vertebral, durante o período de agosto de 2001 a março de 2003. As informações analisadas incluíram: classificação do déficit neurológico nas escalas ASIA (American Spinal Injury Association) e de Frankel, na admissão e alta, tratamento adotado, resultados obtidos e a evolução neurológica. Dos 92 casos, 67 estavam intactos neurologicamente, 16 tinham déficit neurológico completo e, em 8 casos, o déficit era incompleto na admissão. A escala ASIA dos 24 pacientes com déficit neurológico atingiu um índice motor médio de 41,7 (admissão) e 52,06 (alta), com uma diferença de 10,3 pontos. O índice sensitivo foi de 63,4 na admissão e de 82,4 na alta, com diferença de 19 pontos. Dos 38 casos cirúrgicos, 16 tinham déficit neurológico e 22 não. Destes, 21 permaneceram sem déficit com o tratamento cirúrgico e em um caso houve piora neurológica após a cirurgia, com recuperação posterior. Nos 16 casos com dano neurológico verificou-se que 9 melhoraram neurologicamente, 6 mantiveram-se inalterados e 1 piorou. O tratamento conservador foi adotado em 54 casos; entre esses, 4 apresentavam lesão neurológica completa e outros 4, lesão incompleta. Foi indicado quando havia contusão medular e as fraturas eram estáveis, consistindo em colete cervical ou lombar, repouso e analgesia. Seis evoluíram para melhora neurológica e dois permaneceram inalterados. O tratamento realizado (cirúrgico ou conservador) em 24 casos com déficit neurológico levou à melhora de 10,3 pontos no índice motor e de 19 pontos no índice sensitivo.

\section{PALAVRA-CHAVE}

Trauma raquimedular.

\section{ABSTRACT}

Spinal cord lesions: a prospective study of 92 cases

With the objective of evaluating serially the neurological function of spinal cord injured patients, correlating the neurological recovery to the treatment during hospital stay, ninety-two cases treated between August 2001 and March 2003 were analyzed. The variables studied were: type of trauma, neurological deficit in ASIA and Frankel Scales, treatment, results, complications and outcome. Sixtyseven were neurologically intact at admission, 16 had complete deficit and 8 incomplete. ASIA Scale of 24 patients with deficit reached average motor index of 41.7 at admission and 52 when they left hospital. The sensitive average score was 63.4 at admission and 82.4 at the end of hospital stay. From 38 surgical cases, 22 were neurologically normal and 16 had some deficit. Twenty one remained intact after surgery but one presened a transient deficit which recovered later. After surgery nine patients were better, six remained with the same deficit and one became worse. Conservative treatment, consisting on bed rest, cervical or lumbar immobilization and analgesics, was indicated in 54 cases, when the fracture was stable and/or radiological signs of cord contusion were apparent. Among four patients with total deficit and 4 with incomplete lesions, 6 became better and 2 remained inaltered. Surgical stabilization of the spine and did not add any deficit except for one transient neurological worsening in an intact patient.

\section{KEYWORD}

Spinal cord trauma.

\footnotetext{
${ }^{*}$ Professor Titular de Neurocirurgia da Universidade Regional de Blumenau, Chefe do Serviço de Neurocirurgia do Hospital Santa Isabel. ** Acadêmica do Curso de Medicina da Universidade Regional de Blumenau, Bolsista do CNPq-PIBIC.

*** Médico Residente em Neurocirurgia do Hospital Santa Isabel.

**** Neurocirurgião do Serviço de Neurocirurgia do Hospital Santa Isabel.
} 


\section{Introdução}

$\mathrm{O}$ trauma raquimedular (TRM) compreende as lesões dos componentes da coluna vertebral em quaisquer porções: óssea, ligamentar, medular, discal, vascular ou radicular ${ }^{23}$. A prevalência é estimada, nos Estados Unidos, em 5 mil a 10 mil pessoas $/$ ano $^{4}$. Aproximadamente $15 \%$ dos pacientes com trauma de coluna vertebral terão comprometimento neurológico como resultado desse trauma ${ }^{4}$.

O tratamento das fraturas vertebrais inicia-se no atendimento pré-hospitalar, seguindo-se um diagnóstico radiológico precoce e preciso, adequado manuseio da fase aguda, estabelecimento de critérios de instabilidade, tratamento cirúrgico, mobilização precoce e reabilitação $0^{10,15}$.

Em razão desse sério problema, decidiu-se realizar uma avaliação seriada da função neurológica de pacientes com traumatismo raquimedular, observando os mecanismos do trauma, as conseqüências, a opção terapêutica escolhida e a reabilitação neurológica durante a internação.

\section{Casuística e métodos}

Foram analisados, prospectivamente, 92 pacientes internados por causa de trauma raquimedular, no Setor de Neurocirurgia do Hospital Santa Isabel, de agosto de 2001 a março de 2003.

Todos os pacientes tiveram os seguintes dados anotados em protocolo específico, elaborado para o registro de atendimento de trauma raquimedular: idade, sexo, profissão, procedência, causa e nível da lesão, classificação do déficit neurológico na escala ASIA (American Spinal Injury Association) $^{2}$ e de Frankel $^{13}$ na admissão e alta, achado radiológico, tratamento adotado, técnica utilizada, tempo para sua indicação, resultados obtidos, complicações imediatas, evolução e complicações tardias.

A escala ASIA estabeleceu o nível de lesão como correspondente "ao grupo muscular mais caudal com força muscular grau 3 bilateralmente e sensibilidade normal”. No exame sensitivo são testadas apenas as sensibilidades tátil e discriminativa. Foram selecionados grupos musculares-chave, um por nível medular (o mais caudal), que têm seus graus somados para se obter um índice motor cujo valor pode ser, no máximo, 100 pontos. Isso permite a comparação evolutiva dos pacientes, mesmo que essa evolução não implique mudança de nível. Da mesma forma, a sensibilidade é testada em todos os dermátomos e graduada em ausente (zero), diminuída (1) e normal (2). Os resultados são somados, obtendo-se um índice sensitivo, com pontuação máxima de 112 pontos².

A escala de Frankel, outro método de análise da lesão medular, é graduada em cinco níveis: A) déficit motor e sensitivo completo; B) déficit motor completo e sensitivo incompleto; C) déficit sensitivo incompleto e motor incompleto não-funcional; D) déficit sensitivo incompleto e motor incompleto funcional; E) recuperação completa ${ }^{13}$.

Os resultados foram analisados estatisticamente somente em forma descritiva, em virtude de haver um número grande de variáveis para um número baixo de cada uma delas.

\section{Resultados}

A média etária dos 92 pacientes estudados foi de 40,5 anos; 74 (80,4\%) eram do sexo masculino e 18 $(19,5 \%)$ do sexo feminino. O local onde mais ocorreu o acidente foi na cidade de Blumenau, atingindo 60 $(65,2 \%)$ e $32(34,8 \%)$ fora desse município. Destes últimos, 28 vieram encaminhados de outro hospital. O tempo médio de internação foi de 9,2 dias.

Entre as causas do trauma (Gráfico 1), as mais prevalentes foram queda (50\%) e acidente de trânsito (40,2\%). Os segmentos vertebrais mais afetados (Gráfico 2) foram T12-L1 (observado em 34\% dos casos), seguido por C3C7 (constatado em 23\%). Os tipos mais freqüentes de lesões (Gráfico 3) foram as fraturas com acunhamento $(35,8 \%)$ e as fraturas simples (28\%).

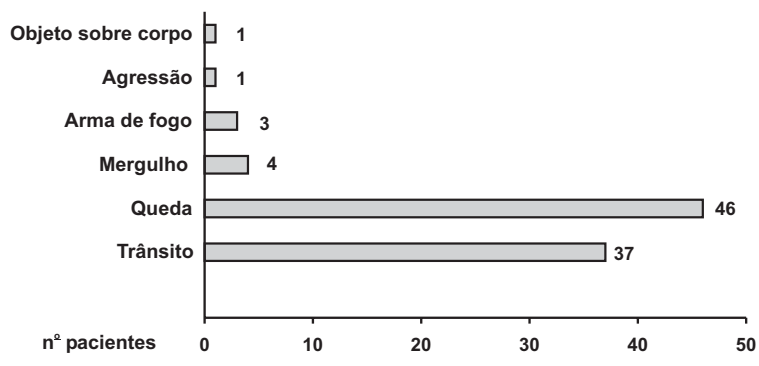

Gráfico 1 - Principais causas de fraturas da coluna vertebral.

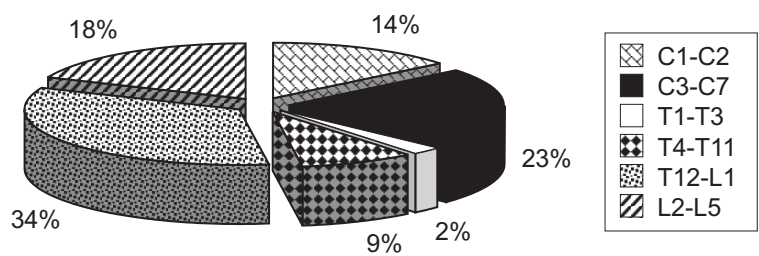

Gráfico 2 - Segmento da coluna vertebral afetado pelo trauma. 


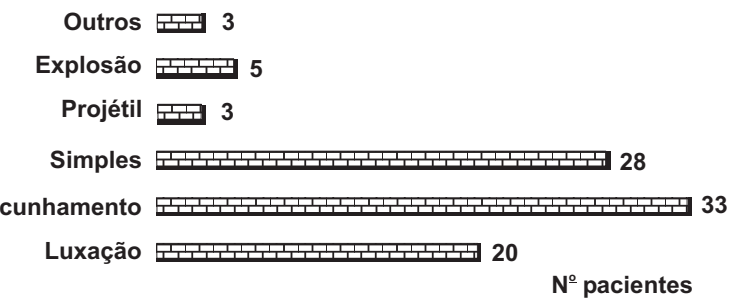

Gráfico 3 - Classificação das fraturas da coluna vertebral.

Dos casos estudados, 24 (26,1\%) manifestavam algum déficit sensitivo-motor na admissão e 67 (72,8\%) apresentaram-se sem déficits. Em um paciente foi impossível realizar o exame neurológico, pois encontrava-se em coma no momento da admissão. O gráfico 4 mostra as conseqüências do trauma nesses pacientes: 10 casos de paraplegia, 6 de tetraplegia, 5 de monoparesia e 3 de tetraparesia.

O exame neurológico foi realizado segundo a escala ASIA, obtendo-se contração anal voluntária em 72 (78,2\%) pacientes; sensibilidade anal em 73 (79,3\%); lesão completa em 16 (17,3\%) e incompleta em 8 (8,6\%). O índice motor médio foi de 41,7 na admissão e 52,06 na alta, com diferença de 10,3 pontos. O índice sensitivo foi de 63,4 na admissão e 82,4 na alta, apresentando uma diferença de 19 pontos (Gráfico 5).
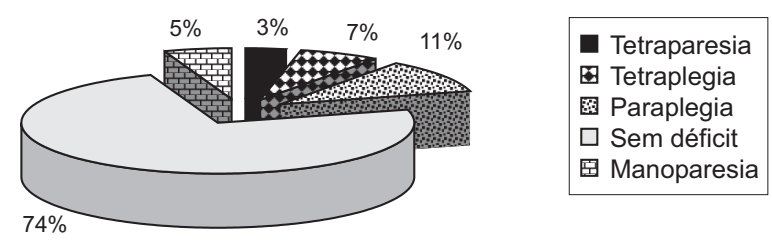

Gráfico 4 - Alterações neurológicas ocasionadas por trauma sobre a coluna vertebral.

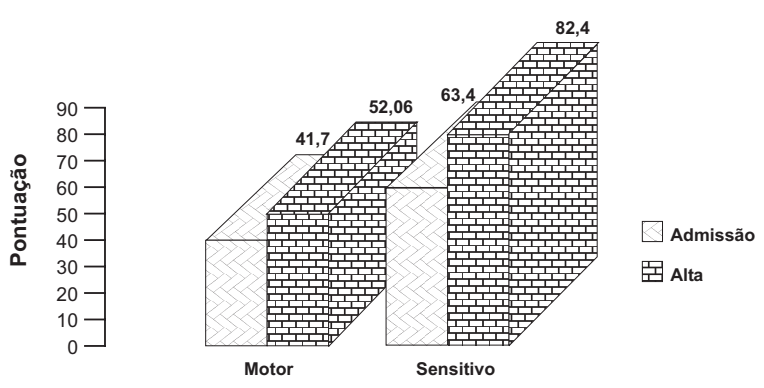

Gráfico 5 - Escala ASIA: avaliação seqüencial do déficit neurológico.
O tratamento adotado foi cirúrgico em 38 (41,3\%) casos e conservador em 54 (58,7\%). As cirurgias realizadas variaram conforme o local da coluna comprometida. Não foram operados especialmente os casos que não apresentavam lesão neurológica e tinham fraturas estáveis, conforme os critérios de Denis ${ }^{8}$.

Dos 24 pacientes com déficit neurológico, 16 foram submetidos ao tratamento cirúrgico, 6 ao tratamento conservador e 2 faleceram precocemente pela gravidade da lesão. Foram tratados de forma conservadora quatro pacientes com contusões medulares e fraturas estáveis (dois casos de fratura de coluna torácica e dois de fratura de coluna cervical), um com ferimento por arma de fogo e um outro que foi transferido para o município de origem, por razões burocráticas.

Dos 16 pacientes com déficit completo na admissão, 12 receberam tratamento cirúrgico e 4 , tratamento clínico. Dos casos cirúrgicos, um obteve melhora neurológica sem mudar de nível na escala de Frankel, um mudou para Frankel B, dois para Frankel D, sete permaneceram iguais e, em um, houve piora (Gráfico 6). Todos os pacientes com déficit completo e tratados clinicamente permaneceram em Frankel A.

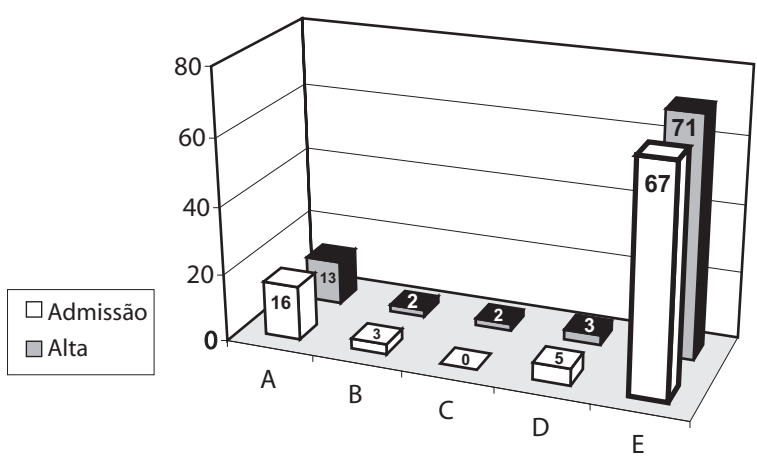

Gráfico 6 - Comparação da evolução neurológica na escala de Frankel.

O tratamento conservador foi o adotado em 54 casos: destes, 33\% apresentavam fraturas no segmento lombar, $28 \%$ no cervical, $22 \%$ no torácico e $17 \%$ na região toracolombar (Gráfico 7). Consistiu em repouso, uso de corticóide ${ }^{6}$, mudança de decúbito a cada duas horas, colchão triangular, colar cervical ou colete lombar de Putti ${ }^{9}$.

O resultado do tratamento instituído nos 24 pacientes com déficit sensitivo-motor foi: recuperação total em 17\%, parcial em 29\%, inalterado em 33\% e piora em 21\% (Gráfico 8). 


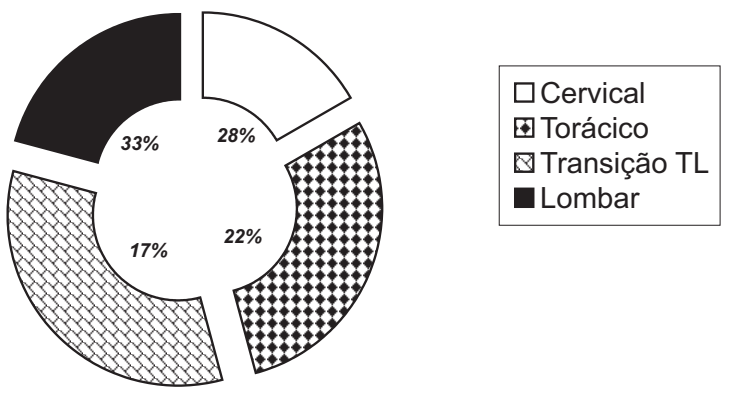

Gráfico 7 - Tratamento conservador aplicado aos segmentos da coluna vertebral.

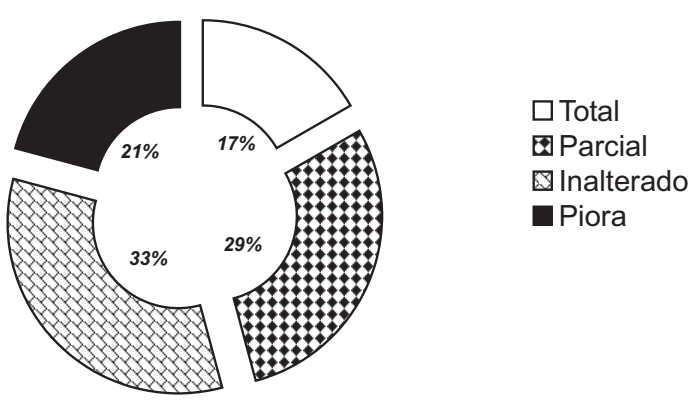

Gráfico 8 - Grau de recuperação neurológica após o tratamento estabelecido.

Os 67 pacientes que não apresentavam déficit na admissão permaneceram inalterados, exceto um caso de piora neurológica transitória.

Dos casos atendidos, $13(14,1 \%)$ precisaram ser internados na Unidade de Terapia Intensiva e 6 (6,5\%) necessitaram de traqueostomia em decorrência de insuficiência respiratória.

As causas dos dez óbitos, sete com lesão neurológica, foram: insuficiência respiratória em sete, meningite bacteriana em um e tromboembolismo pulmonar em dois.

Dos 38 casos cirúrgicos, 16 apresentavam algum déficit neurológico e os outros 22 não. Dos casos sem lesão, 21 permaneceram sem déficit após a cirurgia, um piorou após amarrilha para fixação posterior em C1-C2, com recuperação completa na evolução. Dos 16 que apresentavam lesão, o resultado da cirurgia foi: melhora neurológica em 9; quadro inalterado em 6; piora em 1 , em virtude de insuficiência respiratória seguida de óbito após realização da tração de Crutchfield.
As complicações clínicas observadas nesta casuística foram:

1) relacionadas ao trauma: três $(3,2 \%)$ casos de insuficiência respiratória e um (1,08\%) de derrame pleural;

2) devidas à hospitalização: 12 casos (13\%) de infecção urinária; 8 (8,7\%) de escaras (assim localizadas: sacral, 4; glútea, lombar, trocânter e múltiplas, 3; escara infectada, 1; 8 (8,7\%) de infecção pulmonar; 2 (2,1\%) de tromboembolismo pulmonar; $1(1,08 \%)$ de meningite bacteriana; e $1(1,08 \%)$ de hemorragia digestiva alta;

3) devidas a trauma associado: $8(8,7 \%)$ casos de trauma craniencefálico; 4 (4,3\%) de hematoma extradural; 2 (2,2\%) de pneumotórax; 4 (4,3\%) de politrauma; 1 (1,08\%) de hemotórax; e $1(1,08 \%)$ de hematoma subdural.

Complicações cirúrgicas observadas foram: três casos (7,8\%) de infecção da ferida operatória, um caso de meningite (2,6\%) e outro de piora do déficit neurológico.

Oito pacientes foram reinternados por causa de ruptura do material de implante, deslocamento do local fixado, dor crônica, escaras, empiema pulmonar e volvo gástrico.

\section{Discussão}

O relato deste trabalho, embora com número reduzido de pacientes, justifica-se pelo controle prospectivo realizado e estreito controle neurológico seqüencial dos casos durante o período de internamento hospitalar. Neste grupo houve predominância de sexo masculino $(80,4 \%)$ e adultos com idade média de 40,5 anos, diferente do reportado por Xerez e Maior ${ }^{27}$, que observaram média etária de 23,1 anos.

As causas do trauma mais freqüentes foram queda e acidente de trânsito, reafirmando o observado pela maioria dos estudos ${ }^{20}$, como o de Marczynski e cols. ${ }^{18}$, mas contrariando o estudo, no nosso meio, de Masini e cols. ${ }^{19}$ que verificaram, como causa principal, o acidente de trabalho, seguido dos acidentes de trânsito.

A grande maioria dos casos apresentava-se sem déficit motor no momento da hospitalização (72,8\%), em contraste com o relatado na literatura nacional por Freitas $^{14}$, que observou $86 \%$ dos casos com alguma deficiência motora. Dos pacientes admitidos com déficit neurológico, 66,7\% foram classificados em Frankel A e em B, semelhante ao observado por Xerez e Maior ${ }^{27}$, que encontraram $50 \%$. Todos os pacientes no mesmo grau de Frankel relatados por Botelho e cols. ${ }^{5}$ permaneceram inalterados após a cirurgia, demonstrando a gravidade da lesão inicial. 
A mortalidade em nossa casuística (11\%) encontra-se dentro da variação constatada na literatura de $4,9 \%{ }^{24}$ a $14,28 \%^{1}$ e teve como causas principais insuficiência respiratória por edema medular ascendente, hemorragias digestivas e tromboembolismo pulmonar. Na casuística apresentada, houve um caso de meningite bacteriana como causa de óbito. Em nosso meio, Freitas ${ }^{14}$ observou mortalidade de $24 \%$, provavelmente decorrente de considerável número de casos com politraumatismos entre os seus pacientes, enquanto neste estudo houve apenas 4,3\% de politraumatizados. Aguiar e Machado ${ }^{1}$, em estudo no Brasil, relataram 14,28\% de óbitos em trauma cervical, tendo como causas a insuficiência respiratória e a hemorragia abdominal. Um outro estudo prospectivo e multicêntrico documentou uma mortalidade de 4,9\% durante a hospitalização ${ }^{24}$.

O quadro clínico mostrou maior comprometimento dos membros inferiores, sendo constatado que, quanto mais grave o déficit inicial, pior o prognóstico, conforme relatado na literatura ${ }^{17,22}$. A escala ASIA é um bom método de seguimento para descrever e observar a evolução dos pacientes durante a internação hospitalar, mesmo que o exame neurológico não implique alteração de nível sensitivo e/ou motor ${ }^{17}$.

Cerca de 8\% dos pacientes internados no nosso Serviço apresentaram lesão medular incompleta, contrastando com os dados de Masini e cols. ${ }^{19}$ que relataram déficit parcial em $45 \%$.

A região vertebral mais comumente acometida foi a cervical (37\%), com maior incidência de fraturas com acunhamento (35,8\%) e fraturas simples $(30,4 \%)$, estando em acordo com a literatura, a exemplo de Freitas $^{14}$, que relatou $63 \%$ de casos de lesão cervical, mas com maior número de fraturas com deslocamento (49\%). Outros estudos apóiam a idéia de que o tipo mais comum de lesão vertebral por trauma são as fraturas simples e fraturas-luxação ${ }^{3}$.

Em virtude da variedade de lesões e de achados radiológicos nesses casos, vários tipos de procedimentos são indicados em momentos diferentes ${ }^{8,12,16,26}$, principalmente para beneficiar os pacientes que apresentam desalinhamentos e compressões persistentes ou risco de agravamento devido à instabilidade? .

Cerca de $83 \%$ dos nossos pacientes com alteração no exame neurológico necessitaram de tratamento cirúrgico, diferente da literatura, que mostra uma percentagem de $15 \%$ a $20 \%^{7,24}$.

Quanto à técnica cirúrgica utilizada, a via posterior foi a mais freqüente, principalmente nas grandes instabilidades, uma vez que possibilita facilidade de posicionamento do paciente, adequada via de acesso cirúrgico e abordagem direta da causa de compressão medular $^{7,11,25}$. Já no estudo de Masini e cols. ${ }^{19}$, que envolveu na maioria idosos ${ }^{22}$, a técnica mais utilizada foi a via de acesso anterior ${ }^{21}$.
Os procedimentos cirúrgicos aplicados tiveram bons resultados na medida em que estabilizaram a fratura, diminuíram a dor, permitiram mobilização precoce e ofereceram poucas complicações e riscos cirúrgicos.

O tratamento conservador, aplicado nos casos de fraturas estáveis, também teve seu mérito, pois estabilizou a coluna vertebral mediante o uso de coletes ou colares, diminuiu o desconforto e a dor, além do quadro agudo de edema no local do trauma ${ }^{9}$.

No trabalho de Botelho e cols. ${ }^{5}$ verificam-se como complicações cirúrgicas mais comuns a infecção, a fístula liquórica e o estiramento do plexo braquial. Na nossa casuística, houve apenas três casos de infecção de ferida operatória, um caso de meningite e um caso de piora do déficit neurológico.

\section{Conclusão}

Dos 16 pacientes internados com trauma raquimedular e lesão neurológica completa na admissão, 9 permaneceram no nível A da escala de Frankel após terem sido submetidos a tratamento conservador ou cirúrgico, com estabilização e descompressão medular; quatro pioraram, evoluindo para óbito e, em três, houve melhora, passando um para o nível Frankel B e dois para Frankel D.

Nos oito casos com déficit neurológico incompleto, em seis (75\%) houve melhora da função neurológica após o tratamento adequado, seja conservador ou cirúrgico, e dois (25\%) permaneceram no mesmo nível da escala de Frankel. A escala ASIA mostrou-se eficiente na avaliação neurológica seqüencial.

Quando não havia lesão neurológica na admissão, o tratamento indicado manteve inalterado o exame neurológico, exceto em um caso em que o tratamento proposto acrescentou déficit neurológico temporário.

\section{Referências}

1. AGUIAR LR, MACHADO S: Estabilização cervical posterior utilizando-se placas e parafusos de massa lateral. J Bras Neurocirurg 7:11-21, 1996

2. AMERICAN SPINAL INJURY ASSOCIATION STANDARDS FOR NEUROLOGICAL AND FUNCIONAL CLASSIFICATION OF SPINAL CORD INJURY. Atlanta, GA, American Paralysis Association, 1992.

3. ANDERSON PA, BOHLMAN HH: Anterior decompression and arthrodesis of the cervical spine: long-term motor improvement. Part II. Improvement in complete traumatic quadriplegia. J Bone Joint Surg (Am) 74:6832, 1992 
4. BOER VHT: Trauma raquimedular. In Kruel NF, Araújo PA (eds): Manual de Terapêutica - Cirurgia. Florianópolis, Associação Catarinense de Medicina, 1997, pp 166-71.

5. BOTELHO RV, PEREZ AJ, PALMA MAS, SILVA Jr ASR, FONTOURA EAF: Abordagem lateral extracavitária: utilidade no tratamento do traumatismo raquimedular torácico, toracolombar e lombar. Arq Bras Neurocir (São Paulo) 17:1-10, 1998.

6. BRACKEN MB, SHEPARD MJ, COLLINS WF: Methylprednisolona or naloxone treatment after acute spinal cord injury: 1-year follow-up data. J Neurosurg 76:23-31, 1992.

7. DANISA O, SHAFFREY IS, WHITEHILL JR, WANG J, HANSEN TAS, HANSEN CA, SCHAFFREY ES: Surgical approaches for the correction of unstable thoracolumbar burst fractures: a retrospective analysis of treatment outcome. J Neurosurg 83:977-83, 1995.

8. DENIS F: The Three column spine and its significance in the classification of acute thoracolumbar spinal injuries. Spine 8:817-31, 1983.

9. DENIS F, ARMSTRONG GWD, SEARLS K: Acute thoracolumbar burst fractures in the absence of neurologic deficit. A comparison between operative and nonoperative treatment. Clin Orthop 189:142-9, 1984

10. DONOVAN WH: Operative and nonoperative management of spinal cord injury: a review. Paraplegia 32:375-88, 1994

11. DORAI Z, MORGAN O, COIMBRA C: Titanium cage reconstruction after cervical corpectomy. J Neurosurgery (Spine) 99:3-7, 2003

12. DUH MS, SHEPARD MJ, WILBERGER JE, BRACKEN MB: The effectiveness of surgery on the treatment of acute spinal cord injury and its relation to pharmacological treatment. Neurosurgery 35:240-8, 1994.

13. FRANKEL HL, HANCOCK DO, HYSLOP G, MELZAK J, MICHAELIS LS, UNGAR GH, VERNON JD, WALSH JJ: The value of postural reduction in the initial management of closed injuries of the spine with paraplegia and tetraplegia. Paraplegia 7:179-92, 1969.

14. FREITAS P: Traumatismos raquimedulares agudos: estudo epidemiológico de cem casos consecutivos. J Bras Neurocir 2:1-10, 1990

15. GREVE JMD, CASALIS MEP, BARROS TEP: Diagnóstico e tratamento da lesão da medula espinal. São Paulo, Rocca, 2001, p 400.

16. KRENGEL WF, ANDERSON PA, HENLEY MB: Early stabilization and descompression for incomplete paraplegia due to a thoracic-level spinal cord injury. J Spine 18:2080-7, 1993.
17. MCDONALD JH, BECKER D, SADOWSKY CL, JANE JÁ, CONTURO TE, SCHULTZ LM: Late recovery following spinal cord injury. J Neurosurg (Spine) 97: 25265, 2002.

18. MARCZYNSKI W, KROCZAK S, BARANSKI M: Fractures of thoracic and lumbar spine; treatment and follow up. Ann Transplant 4:46-8, 1999.

19. MASINI M, ZAHRAAH, BARROSO CBRB: Síndrome de lesão centro medular cervical traumática (Schneider). Revisão de 50 casos. J Bras Neurocirurg 7:44-51, 1996.

20. PICKETT W, SIMPSON K, WALKER J, BRISON RJ: Traumatic spinal cord injury in Ontario, Canada. J Trauma 55:1070-6, 2003.

21. SCHNEE CLS, ANSELL LV: Selection criteria and outcome of operative aprroaches thoracolumbar burst fractures with and without neural deficit. J Neurosurg 86:48-55, 1997.

22. SCIVOLETTO G, MORGANTI B, DITUNNO P, DITUNNO JF, MOLINARI M: Effects on age on spinal cord lesion patients rehabilitation. Spinal Cord 41:457-64, 2003.

23. SILVEIRA PR: Trauma raquimedular: diagnóstico e tratamento nas emergências. Bras Med 78:17-37, 2000.

24. SUMAS ME, NARAYAN RK: Head injury. In Grossman RG, Loftus CM (eds): Principles of neurosurgery. Ed 2. Philadelphia, Lippincott- Raven, 1999, pp 157-9.

25. SUTTERLIN CE, MCAFEE PC, WARDE KE, REY RMJ, FAREY ID: A biomechanical evaluation of cervical spinal stabilization methods in a bovine model. Spine 13:797802, 1988.

26. WILLEN J, DAHLLOF A, NORDWALL A: Paraplegia in unstable thoracolumbar injuries: a study of conservative and operative treatment regarding neurological improvement and rehabilitation. Scand J Rehabil Med Suppl 9:195-205, 1983.

27. XEREZ DR, MAIOR IL: Seleção de amostra de portadores de lesão medular completa. Med Reabil 44:710, 1996.

Original recebido em setembro de 2004

Aceito para publicação em novembro de 2004

\author{
Endereço para correspondência: \\ Luís Renato Mello \\ Rua Ferdinando Schadrack, 29 \\ CEP 89050-370 - Blumenau, SC \\ E-mail: lrenatomello@terra.com.br
}

